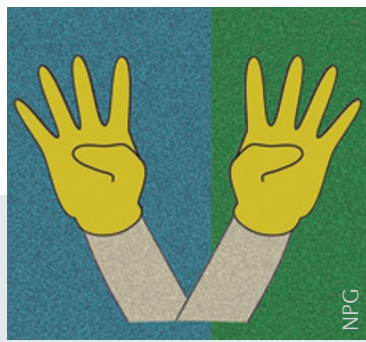

\title{
Lyrica survives patent challenge
}

Pfizer has successfully fended off a patent challenge by a group of generics companies that wanted to market their own version of Lyrica (pregabalin). The drug, which is used to treat seizures and pain, had a revenue of US\$525 million in the United States in the fourth quarter of 2013.

The collective of generics companies - which included Teva, Lupin, Actavis and Cobalt - challenged a key claim in one of Lyrica's patents (US 6197819) that described 4-amino-3-(2-methylpropyl) butanoic acid. Of note, Lyrica is the (S)-enantiomer of this compound. The generics challengers asserted that this claim covered only the racemate, not the (S)-enantiomer. In this way, their proposed products, which would contain "non-racemic mixtures", would not infringe on the patent.

But Pfizer said that the compound described in the claim was not limited to the racemate, so it could be any isomeric form.
The US Appeals Court noted that, elsewhere in the patent, Pfizer had used specific terminology $((R),(S)$, racemic and so on) to specify when it referred to a specific isomeric form. In the Court's opinion, there was no basis to support the assertion of the generics companies that the absence of an $(R)$ or $(S)$ prefix in the claim meant that it referred to the racemate.

So because the key claim encompassed the compound in any enantiomeric form, the products proposed by the generics companies would have infringed on Lyrica's patent.

The Court also found, in Pfizer's favour, that Lyrica was sufficiently described in patents to enable others to make it, and that Lyrica was not obvious over other compounds with a similar structure and anticonvulsant activity that were described in the prior art. The ' 819 patent expires in December 2018.

Pfizer versus Teva et al.: http://www.cafc.uscourts.gov/images/stories/opinionsorders/12-1576.Opinion.2-3-2014.1.PDF

\section{US Patent Office issues guidelines on natural product patent eligibility}

The two recent high-profile US Supreme Court decisions on patent eligibility Association for Molecular Pathology versus Myriad (see Nature Rev. Drug Discov. 12, 570-571;2013) and Mayo versus Prometheus (see Nature Rev. Drug Discov. 11, 344; 2012) changed the rules on what type of inventions were eligible for patent protection. Namely, the long-standing precedent that products that are isolated from natural sources could be patented was thrown out, and certain methods of determining optimal drug doses were also deemed to be patent eligible.
In response to these changes, the US Patent and Trademark Office has now issued guidance to help patent examiners (and indeed patentees) determine whether applications are patent eligible.

The publication details several examples of hypothetical patent applications and whether they should be permissible. For instance, if a cancer-combating compound was isolated and purified from plant leaves, it would not be eligible because it would not be substantially different from the natural product that is present in the leaves. However, a derivative that was structurally and functionally different could be patent eligible, as could a method of using the isolated purified natural product to treat colon cancer.
Whether the new guidelines mean that a greater proportion of applications are rejected - and potentially appealed remains to be seen. But it has been noted that several historically patented products, such as adrenaline and streptomycin, would fail to meet the new eligibility guidelines.

US Patent and Trademark Office guidelines: http://www.uspto.gov/patents/law/exam/myriad-mayo grs. pdf; http://www.uspto.gov/patents/law/exam/myriadmayo guidance.pdf

Charlotte Harrison

PATENT ADVISORS

Daniel M. Becker: Dechert, Mountain View, CA, USA Luke Kempton: Wragge \& Co., London, UK. Leslie Meyer-Leon: IP Legal Strategies, Boston, MA, USA. George W. Schlich: Schlich \& Co., London, UK. John A. Tessensohn: Shusaku Yamamoto, Osaka, Japan. Philip Webber: Dehns, London, UK.

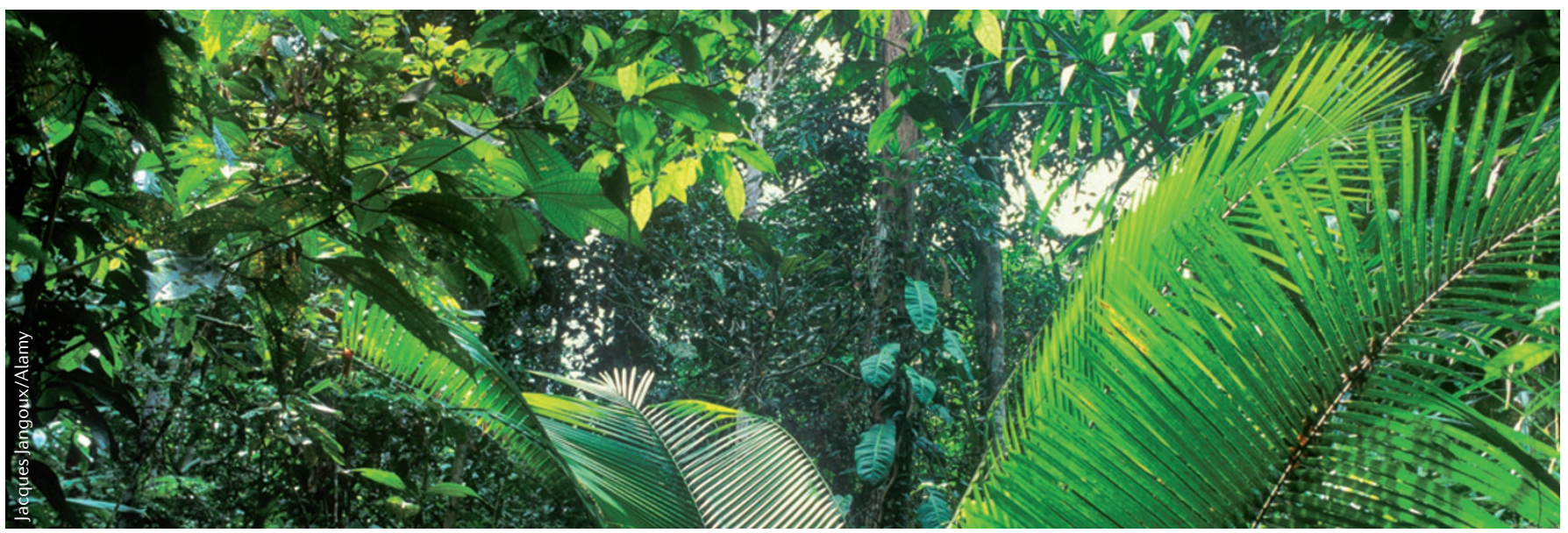

